\title{
925.
}

\section{ON WARING'S FORMULA FOR THE SUM OF THE $m$ th POWERS OF THE ROOTS OF AN EQUATION.}

[From the Messenger of Mathematics, vol. XxI. (1892), pp. 133-137.]

The formula in question, Prob. I. of Waring's Meditationes Algebraica, Cambridge, 1782, making therein a slight change of notation, is as follows: viz. the equation being

then we have

$$
x^{n}+b x^{n-1}+c x^{n-2}+d x^{n-3}+\ldots=0,
$$

$$
\begin{aligned}
& (-)^{m} S_{m}= \\
& \begin{array}{rlr} 
& b^{m} & +m \cdot h \\
-m c & b^{m-2} & -m \cdot m-6 \cdot c f \\
+m d & b^{m-3} & -m \cdot m-6 \cdot d e
\end{array} b^{m-7} \\
& \left.-m e\}_{b^{m-4}} \quad+\frac{1}{2} m \cdot m-5 \cdot m-6 \cdot c^{2} d\right) \\
& +\frac{1}{2} m \cdot m-3 \cdot c^{2} \int^{b^{m-4}} \\
& +m f\}_{b^{m-5}}+m \cdot m-7 . c g \\
& -m \cdot m-4 . c d\}^{b^{m-5}}+m \cdot m-7 \cdot d f \\
& \left.\left.\begin{array}{rr}
-m \cdot g \\
+m \cdot m-5 \cdot c e \\
+\frac{1}{2} m \cdot m-5 \cdot d^{2} \\
-\frac{1}{6} m \cdot m-4 \cdot m-5 \cdot c^{3}
\end{array}\right\} \begin{array}{r}
+\frac{1}{2} m \cdot m-7 \cdot e^{2} \\
b^{m-6}
\end{array} \begin{array}{r}
b^{m-8} \\
+\frac{1}{24} m \cdot m-5 \cdot m-6 \cdot m-7 \cdot c^{4}
\end{array}\right\} \\
& \text { +\&c. }
\end{aligned}
$$

where, reckoning the weights of $b, c, d, e, \ldots$ as $1,2,3,4, \ldots$, respectively, the several terms are all the terms of the weight $m$, or (what is the same thing) in the 
coefficient of $b^{m-\theta}$ we have all the combinations of $c, d, e, \ldots$, (or say all the non-unitary combinations) of the weight $\theta$, and where the numerical coefficient of

is

$$
b^{m-\theta} c^{\mathrm{c}} d^{\mathrm{d}} e^{\mathrm{e}} \ldots(\mathrm{c}+\mathrm{d}+\mathrm{e}+\ldots=\theta)
$$

$$
=(-)^{\mathrm{c}+\mathrm{e}+\mathrm{g}+\ldots} \frac{m \cdot m-(\theta-\delta+1) \cdot m-(\theta-\delta+2) \ldots m-(\theta-1)}{\Pi \mathrm{c} \cdot \Pi \mathrm{d} \cdot \Pi \mathrm{e} \ldots} \text {. }
$$

Thus for the term $b^{m-8} c^{2} e^{1}, \theta=8 ; c, d, e=2,4,1$ respectively (the other exponents each vanishing), and the coefficient is

$$
(-)^{3} \frac{m \cdot m-6 \cdot m-7}{1 \cdot 2 \cdot 1},=-\frac{1}{2} m \cdot m-6 \cdot m-7,
$$

as above; and so in other cases.

For the MacMahon form

$$
1+b x+\frac{c x^{2}}{1.2}+\ldots=(1-\alpha x)(1-\beta x) \ldots
$$

or say

$$
y^{n}+\frac{b}{1} y^{n-1}+\frac{c}{1.2} y^{n-2}+\ldots=(y-\alpha)(y-\beta) \ldots
$$

we must for $b, c, d, \ldots$, write $b, \frac{c}{1.2}, \frac{d}{1.2 .3}, \ldots$ respectively: we thus have

$$
\begin{aligned}
(-)^{m} \cdot S_{m}= & b^{m} \\
& -m \frac{c}{1 \cdot 2} \\
& +m \frac{d}{1 \cdot 2 \cdot 3} \\
& -m \frac{e}{1 \cdot 2 \cdot 3 \cdot 4} \\
& +\frac{1}{2} m \cdot m-3\left(\frac{c}{1 \cdot 2}\right)^{2} \\
& +\& c .,
\end{aligned}
$$

or say

$$
\begin{aligned}
& (-)^{m} \Pi(m-1) S_{m}=\Pi(m-1) \\
& -\mathrm{II} m \frac{c}{1.2} \\
& +\Pi m \frac{d}{1.2 .3} \\
& \left.\begin{array}{l}
-\Pi m \frac{e}{1.2 .3 .4} \\
+\Pi m \frac{m-3}{2}\left(\frac{c}{1.2}\right)^{2}
\end{array}\right\} \\
& +\& c \text {. }
\end{aligned}
$$


the numerical coefficient of

being

$$
b^{m-\theta} c^{\mathrm{e}} d^{\mathrm{d}} e^{\mathrm{e}} \ldots(\mathrm{c}+\mathrm{d}+\mathrm{e}+\ldots=\theta)
$$

$$
(-)^{\mathrm{c}+\mathrm{e}+\mathrm{g}+\ldots} \frac{\Pi m \cdot m-(\theta-\delta+1) \cdot m-(\theta-\delta+2) \ldots m-(\theta-1)}{\Pi \mathrm{c} \cdot \Pi \mathrm{d} \cdot \Pi \mathrm{e} \ldots(\Pi 2)^{\mathrm{c}}(\Pi 3)^{\mathrm{d}}(\Pi 4)^{\mathrm{e}} \ldots} .
$$

It is convenient to write down the literal terms in alphabetical order $(A O)$, calculating and affixing to each term the proper numerical coefficient; thus taking

$$
1+b x+c \frac{x^{2}}{1.2}+\ldots=(1-\alpha x)(1-\beta x)(1-\gamma x) \ldots,
$$

we find

$$
\begin{aligned}
& -120 S_{6}=g \quad \cdot \quad 1 \\
& \text { bf . }-6 \\
& \text { ce . }-15 \\
& d^{2} \cdot-10 \\
& b^{2} e .+30 \\
& b c d .+120 \\
& c^{3} .+30 \\
& b^{3} d \text {. }-120 \\
& b^{2} c^{2} \cdot-270 \\
& b^{4} c .+360 \\
& b^{6} \quad-120 \\
& \pm 541
\end{aligned}
$$

this expression, as representing the value of the non-unitary function $S_{6}$, being in fact a seminvariant.

It is to be remarked that the foregoing expression for the sum of the $m$ th powers of the roots of the equation

$$
x^{n}+b x^{n-1}+c x^{n-2}+\ldots=0
$$

is, in fact, the series, for $x^{m}$ continued so far only as the exponent of $b$ is not negative: see as to this Note XI. of Lagrange's Équations Numériques. For the à posteriori verification, observe that we have

$$
x+b+\frac{c}{x}+\frac{d}{x^{2}}+\ldots=0
$$

or writing for a moment $u=-b$, say this is

$$
x=u+f x,
$$


where

$$
f x=-\frac{c}{x}-\frac{d}{x^{2}}-\frac{e}{x^{3}}-\& c
$$

Hence, by Lagrange's theorem,

$$
\begin{aligned}
x^{m}= & u^{m} \\
& -m u^{m-1}\left(\frac{c}{u}+\frac{d}{u^{2}}+\frac{e}{u^{3}}+\ldots\right) \\
& +\left\{m u^{m-1}\left(\frac{c}{u}+\frac{d}{u^{2}}+\frac{e}{u^{3}}+\ldots\right)^{2}\right\}^{\prime} \frac{1}{1.2} \\
& -\left\{m u^{m-1}\left(\frac{c}{u}+\frac{d}{u^{2}}+\frac{e}{u^{3}}+\ldots\right)^{3}\right\}^{\prime \prime} \frac{1}{1.2 .3} \\
& + \text { \&c. }
\end{aligned}
$$

where the accents denote differentiations in regard to $u$. This is

$$
\begin{aligned}
= & u^{m} \\
& -m\left\{c u^{m-2}+d u^{m-3}+e u^{m-4}+f u^{m-5}+g u^{m-6}+\ldots\right\} \\
& +\frac{1}{2} m\left\{(m-3) c^{2} u^{m-4}+(m-4) 2 c d u^{m-5}+(m-5)\left(d^{2}+2 c e\right) u^{m-6}+\ldots\right\} \\
& -\frac{1}{6} m\left\{(m-4)(m-5) c^{3} u^{m-3}+\ldots\right\} \\
& +\& c . \\
= & u^{m} \\
& +u^{m-2} \cdot-m c \\
& +u^{m-3} \cdot-m d \\
& +u^{m-4} \cdot-m e+\frac{1}{2} m \cdot m-3 \cdot c^{2} \\
& +u^{m-5} \cdot-m f+\frac{1}{2} m \cdot m-4 \cdot 2 c d \\
& +u^{m-6} \cdot-m g+\frac{1}{2} m \cdot m-5 \cdot\left(d^{2}+2 c e\right)-\frac{1}{6} m \cdot m-4 \cdot m-5 \cdot c^{3} \\
& +\& c .,
\end{aligned}
$$

which, putting therein $u=-b$ and multiplying each side by $(-)^{m}$, is the beforementioned formula for $(-)^{m} \mathrm{Sa}^{m}$ : in that formula the series being continued only so far as the exponent of $b$ is not negative.

I notice also that we cannot easily, by means of the known formula

$$
S \alpha^{m} \beta^{p}=S \alpha^{m} \cdot S a^{p}-S \alpha^{m+p},
$$

deduce an expression for $S \alpha^{m} \beta^{p}$ : in fact, forming the product of the series for $S \alpha^{m}$, $S \alpha^{p}$ respectively, this product is identically equal to the series for $S \alpha^{m+p}$, or we seem to obtain $0=S \alpha^{m} S \alpha^{p}-S \alpha^{m+p}$; to obtain the correct formula, we have to take each of the three series only so far as the exponent of $b$ therein respectively is not negative: and it is not easy to see how the resulting formula is to be expressed. 